\title{
Concept esthétique et non invasif pour l'implantation des secteurs antérieurs des maxillaires
}

\section{Minimally Invasive Esthetic Concept of Anterior Region}

\section{MOTS-CLEFS :}

- Zone esthétique, déficit osseux, position tridimensionnelle de l'épaule de l'implant, couronne ò pilier intégré (PCI)

\section{KEYWORDS:}

- Esthetic zone, bone deficiency, 3-D Implant shoulder position, integrated abutment crown.

\section{Résumé}

La clé d'une réussite esthétique réside dans le placement de l'implant dans une position tridimensionnelle bien précise. Le déficit osseux de la zone maxillaire antérieure est un phénomène très fréquent qui va rendre cet objectif inaccessible sans augmentation pré-ou per-opératoire de la crête.

L'expérience clinique dans la zone antérieure déficitaire nous a donné des résultats esthétiques durables et très satisfaisants sans recourir à la chirurgie lourde de gestion de crête. Cette dernière est basée sur les points suivants : la morphologie et l'orientation de l'implant, l'approche chirurgicale adéquate avec une procédure manuelle de manipulation osseuse et la restauration prothétique notamment avec le pilier à couronne intégrée. Tous ces facteurs interagissent pour atteindre notre objectif, que nous avons nommé le Concept esthétique et non invasif de la zone Maxillaire antérieure.

\section{Abstract}

The key for a successful aesthetic result of the anterior zone is in the placement of the implant in a precise three-dimensional position. The bone defect in aesthetic zone, a very common phenomenon, makes this goal inaccessible without pre- or operative ridge management.

The clinical experience obtained with a very satisfying long lasting aesthetic results of different defected aesthetic zone without going through the major surgical procedure of ridge management is based on implant morphology and orientation, appropriate surgical approach with manual surgical procedure of bone handling and manipulation and finally prosthetic restoration with Integrated Abutment Crown.

All these factors interact together to achieve our goal with with what we call Minimally Invasive Esthetic Concept of the anterior zone of Maxilla.
AOS 2014;267:40-48 DOI: $10.1051 /$ aos/2014107 (C) EDP Sciences 2014

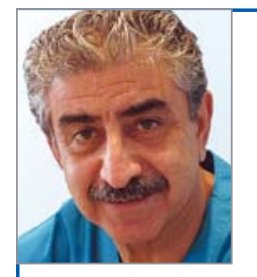

- Kadhim AL HIMDANI

French Center for Dental Implant, Émirats Arabes Unis - Sharjah

Bouhaira Cornich - Cornich Plaza-1- Cabinet: (601),

PO Box $6 G_{167}$ (E.A.U).

dhimdani@yahoo.com 


\section{INTRODUCTION}

Dans notre pratique quotidienne, la pose d'un implant dentaire dans la zone antérieure du maxillaire est considérée comme un réel défi professionnel pour deux raisons : l'augmentation des exigences des patients pour l'obtention de résultats esthétiques durables dans une région où plusieurs facteurs locaux sont susceptibles de compromettre le résultat final [1, $2,4]$; de plus, la particularité et/ou la difficulté de la mise en place immédiate de l'implant avec ou sans restauration (qui est la procédure la plus sollicitée) rendent cette tâche plus difficile encore.

En effet, le consensus international insiste sur le fait que la clé d'une réussite esthétique, quel que soit le système d'implant utilisé, est le placement de l'épaulement de l'implant dans une position tridimensionnelle bien précise exigeant ainsi un volume osseux considérable [2] (fig. 1).
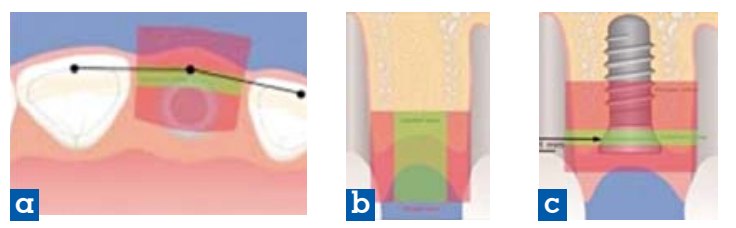

$\triangle$ Figure 1:

Position tridimensionnelle de l'épaule de l'implant. (a) Oro-facial. (b) Mésio-distal. (c) Apico-coronal [2].

Malheureusement, le déficit osseux de la zone antérieure du maxillaire est un phénomène très fréquent, qui va rendre cet objectif inaccessible ou difficile à atteindre sans augmentation pré- ou peropératoire de la crête.

L'objectif de cette série de cas est de partager notre expérience clinique au cours de laquelle nous avons obtenu des résultats esthétiques durables et très satisfaisants pour différentes situations critiques de déficit osseux de la zone esthétique. Ceci sans passer par des procédures de gestion de la crête alvéolaire qui sont habituellement plus complexes, plus longues, plus douloureuses et plus coûteuses, telles que les greffes osseuses, la préservation de l'alvéole et la distraction osseuse.

Pour cela, une évaluation approfondie des tissus mous et durs ainsi que les paramètres esthétiques doivent être pris en considération afin d'avoir un plan de traitement approprié et éviter ainsi toute complication d'ordre esthétique.

L'hygiène buccodentaire et l'état parodontal devront être soigneusement évalués cliniquement et radiologiquement [7]. En cas d'antécédent de parodontite chronique, il faut informer le patient du risque éventuel d'un triangle noir interdentaire.

\section{ÉVALUATION DES TISSUS MOUS}

Le biotype gingival doit être évalué car il joue un rôle important dans la récession gingivale post-chirurgicale $[8,9]$.

\section{Biotype gingival}

Des études ont montré qu'il existe des différences d'épaisseur des tissus mous [10].

Le biotype mince a une architecture gingivale très échancrée et une épaisseur des tissus mous très réduite par rapport au biotype gingival épais qui comporte généralement un contour émoussé des papilles $[2,11,12]$.

Pour les patients ayant un biotype gingival mince, la pose d'implants dans une position légèrement plus apicale et palatine permettra de réduire le risque de récession gingivale et de prévenir l'ombre du titane qui peut apparaitre à travers le tissu gingival [2]. Ainsi, un volume adéquat de tissus mous offre un bon profil d'émergence et sert à masquer l'implant métallique sous-jacent.

Chez les patients à haut risque ayant une épaisseur gingivale minime (biotype gingival mince), l'augmentation des tissus mous sera une solution plus prudente. Sinon, les patients doivent être informés de la possibilité d'une récession gingivale postopératoire avec toutes ses conséquences inesthétiques. Parfois même, une prothèse conventionnelle peut être conseillée [2].

\section{Évaluation esthétique}

Le contour des lèvres est un autre paramètre qui doit être pris en considération. Les patients présentant une ligne de sourire haute avec un biotype gingival mince sont considérés comme des patients à haut risque dont la planification de traitement doit être très sévère.

\section{ÉVALUATION DU TISSU DUR}

L'évaluation de la structure osseuse du site édenté doit être déterminée après une étude clinique et radiographique soigneuse. Nous devons être prudents devant l'aspect clinique d'une crête alvéolaire d'épaisseur considérable qui peut être composée principalement de tissu mou (biotype gingival épais) (fig. 2).
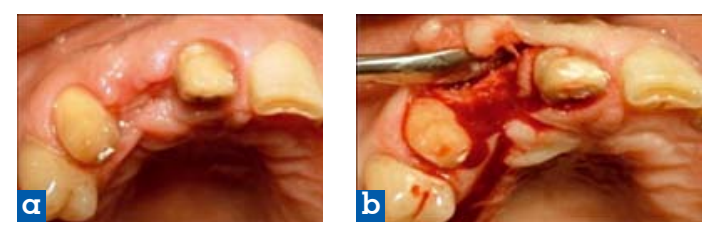

$\triangle$ Figure 2 :

(a-b) : Édentement (12) avant et après lambeau. 
Pour cela, une image radiographique préopératoire en trois dimensions est la seule mesure précise pour évaluer le site osseux édenté même s'il s'agit d'un édentement unitaire. Sinon, un lambeau d'épaisseur totale doit être soulevé (fig. 2b) pour apprécier en vision directe l'épaisseur et la forme de la crête alvéolaire afin de pouvoir placer l'implant dans la bonne position en évitant ainsi la fenestration osseuse de la table externe.

L'épaisseur de l'os vestibulaire influence de façon significative le degré de la résorption verticale. Ferrus et al. 2010 [14] ont évalué que dans les sites avec une crête osseuse vestibulaire d'épaisseur supérieure à $1 \mathrm{~mm}$, la hauteur moyenne de résorption verticale de cet os était faible $(0,4$ à $1,3 \mathrm{~mm})$. Pour les sites ayant une paroi mince vestibulaire inférieure à $1 \mathrm{~mm}$, il y avait une importante perte de la dimension verticale $(1,2-2,1 \mathrm{~mm})$.
Pour fournir un bon support pour les tissus mous et éviter la résorption osseuse, l'épaisseur idéale de l'os autour de l'implant doit mesurer de 1,5 à $2 \mathrm{~mm}$ $[15,18,19]$.

Après l'avulsion d'une dent chez presque $90 \%$ des patients, la paroi vestibulaire de l'alvéole disparaîtra dans les premiers six mois [15, 16, 17] (fig. 3).
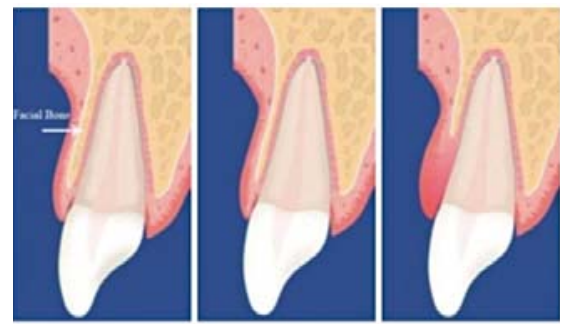

Très rarement Très fréquemment Fréquemment

$\triangle$ Figure 3 :

Étude des parois, paroi alvéolaire et vestibulaire [3].

\section{SERIE DE CAS CLINIQUES}

Nous présentons maintenant notre expérience clinique des cinq dernières années face à ces problèmes quotidiens de déficit de l'os alvéolaire dans la zone esthétique et les solutions adoptées pour obtenir un résultat esthétique optimal sans traumatiser nos patients avec des procédures d'augmentation de la crête grâce au Concept Esthétique et non Invasif de la zone Maxillaire antérieure.
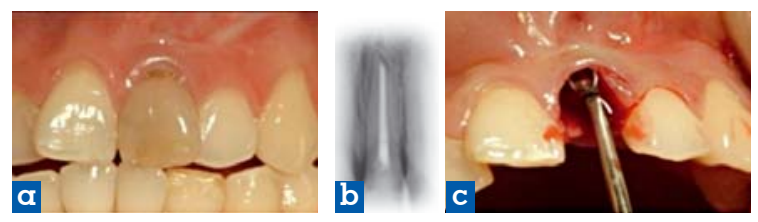

\section{CAS 1 (fig. 4)}

Femme de 24 ans. L'incisive centrale supérieure gauche (21) présente une lésion radiculaire avec biotype gingival mince et une absence totale de la table externe.
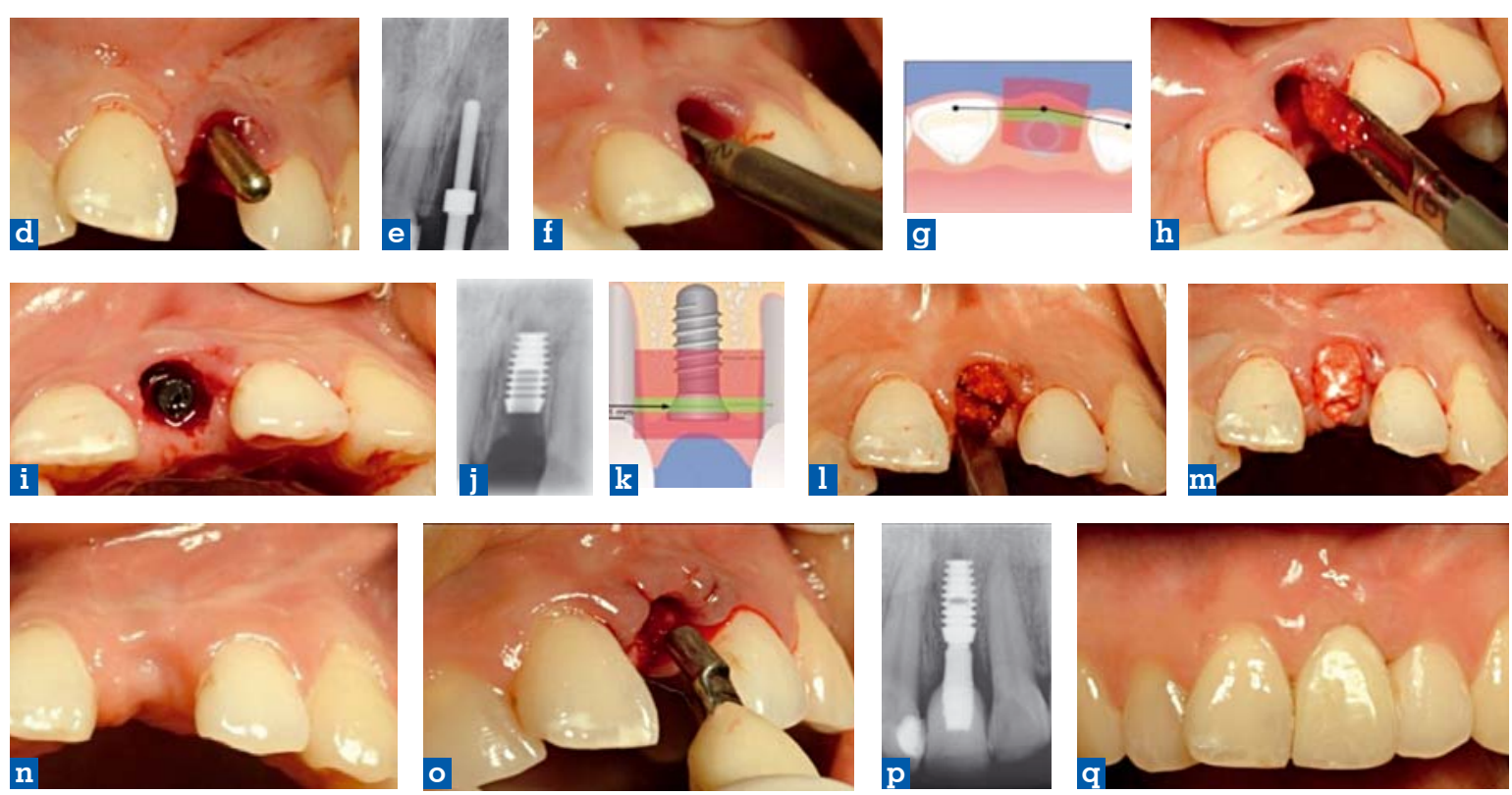

$\triangle$ Figure 4 :

(a et b) Aspect clinique et radiologique de la (21) avec biotype gingival mince. (c) Absence totale de la table externe. (d) Orientation palatine.

(e) Radio contrôle. (f et g) Alésoir manuel 2.5 très vestibulaire.

(h) Récolte d'os autogène. (i, j, k) Position finale de l'implant en dehors des règles tridimensionnelles. (l) Greffe d'os autogène $(\mathrm{m})$ recouverte par une membrane de collagène $(\mathrm{n}) 4$ mois après. (o et p) Restauration avec un pilier à couronne intégrée. (q) Résultat final après 1 an.

\section{Gestion}

Implant court et étroit placé profondément (3-4 mm subcrestal) avec appréciation manuelle de l'os. (f et $\mathrm{h}$ ) + Orientation adaptée de l'implant $(\mathrm{d}-\mathrm{i}-\mathrm{j})+$ Greffe osseuse autogène $(\mathrm{l})+$ Chirurgie en 2 temps-[mise en nourrice] $(\mathrm{n})+$ Pilier à couronne intégrée PCI (o). 

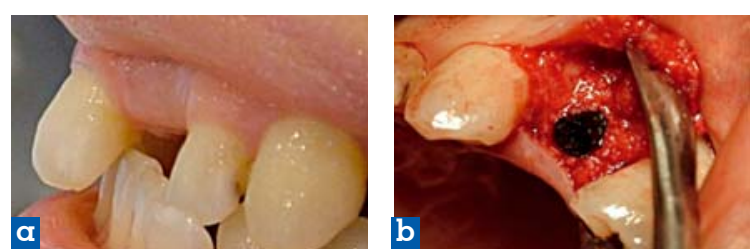

$\triangle$ Figure 5 :

(a) Pré-opératoire. (b) Implant avec expansion et greffe autogène. (c et d) Clinique et radio 2 ans postopératoire.
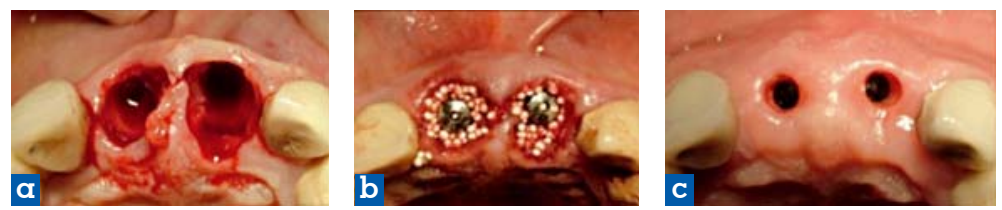

(a) Absence totale de la tal (b) Implant (chirurgie en deux temps) + greffe de l'os. (c) Aspect clinique 3 mois après. (d) Résultat final avec couronnes individuelles 1 an après.
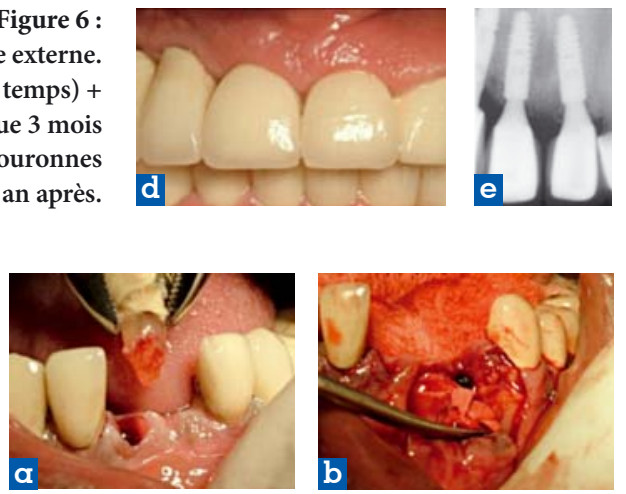

Figure 7 :

(a) La 41 présentant une accumulation de dépôts durs (tartre) et une alvéolyse atteignant le tiers apical. (b) Implant (32) en place, déhiscence osseuse comblée par os autogène. (c et d) Aspect et radiologie

2 ans après restauration prothétique.
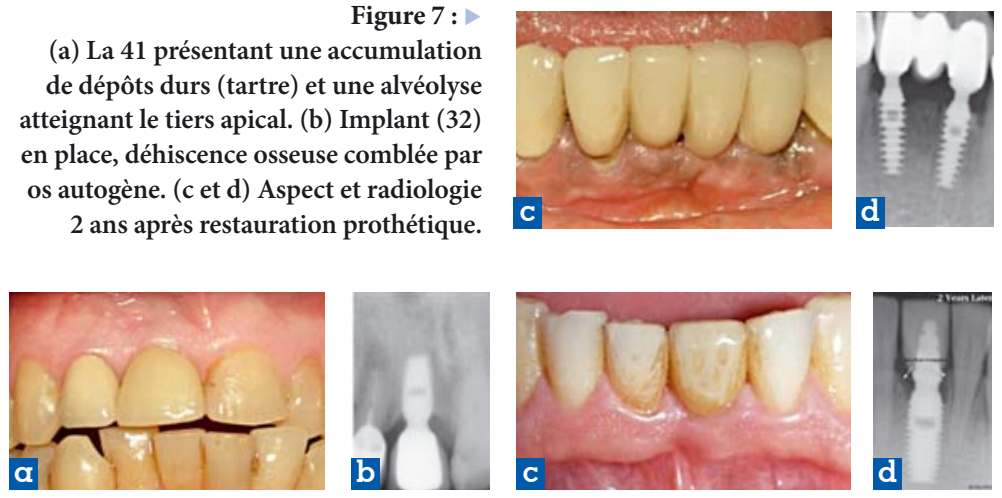

Figure 8 :

(a et b) La (11) 42 mois après restauration avec un déficit apico-coronal. (c et d) La (31) deux ans après restauration avec un déficit apico-coronal et mésio-distal.

\section{CAS 2 (fig. 5)}

Femme de 37 ans. Absence de (21) avec supracculsion antérieure. Déficit vestibulo-palatin de la crête.

\section{Gestion}

Implant étroit 3,5 $\mathrm{mm}$ placé profondément $(2 \mathrm{~mm}$ subcrestal) avec appréciation manuelle de l'os + Orientation de l'implant guidé par l'os disponible + Greffe osseuse autogène + Expansion de la crête alvéolaire + 2 temps chirurgicaux.

\section{$\mathrm{CAS}_{3}$ (fig. 6)}

Homme de 60 ans. Fractures radiculaires (11-21) restaurées avec couronnes et inlay cores.

Absence totale de la table externe.

\section{Gestion}

Implant étroit 3,5 $\mathrm{mm}$ placé immédiatement $(2 \mathrm{~mm}$ subcrestal) avec appréciation manuelle de l'os + Orientation adaptée de l'implant + Greffe de l'os synthétique +1 temps chirurgical.

\section{CAS 4 (fig. 7)}

Homme de 57 ans. Antécédent de parodontite chronique (32-41), avec déficit osseux (oro-facial et apico-coronaire) et absence de la table externe.

\section{Gestion}

Nombre réduit d'implant, étroit placé profondément (2 $\mathrm{mm}$ subcrestal) avec M.A.O.A.O + Orientation adaptée l'implant + Greffon osseux autogène +2 temps chirurgicaux.

\section{Discussion}

Cette série de cas clinique illustre notre concept pour résoudre ces problèmes de déficits osseux de la zone esthétique avec le minimum de traumatisme et d'interventions chirurgicales. C'est le Concept Esthétique et non Invasif de la zone Maxillaire antérieure qui est basé sur les quatre principes essentiels de l'implantologie moderne (fig. 9).

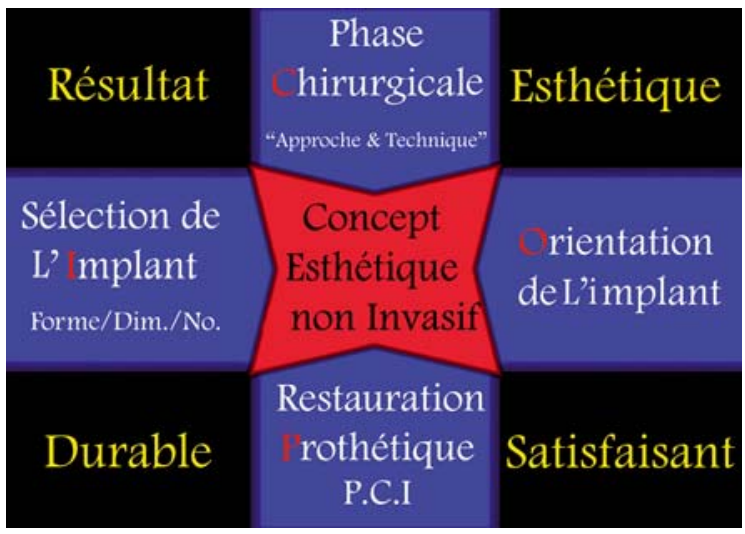




\section{SÉLECTION DE L'IMPLANT}

Une sélection de l'implant en termes de forme, taille, et nombre est capitale pour l'obtention d'un résultat esthétique durable.

\section{Forme de l'implant}

\section{Switch Platform (fig. 10)}

Le concept de Switch Platform signifie que le pilier est de diamètre inférieur à celui du col de l'implant. De ce fait, le périmètre de la jonction implant-pilier (JIP) où se trouve le microgap sera déplacé vers l'intérieur, réduisant ainsi les risques de résorption osseuse de la crête grâce à une amélioration de la qualité du tissu mou (Biotype Gingival) [23, 25-29]. L'augmentation de l'épaisseur de la muqueuse péri-implantaire va être un élément fondamental pour masquer l'aspect grisâtre de l'implant.

\section{Épaulement oblique (fig. 10b)}

L'épaulement oblique de l'implant offre davantage de place pour la croissance osseuse sur l'implant donnant ainsi plus de soutien aux papilles interdentaires.

\section{Design des spires (Design du Plateau ) (fig. 11)}

Le concept de cette morphologie permet d'augmenter la surface de contact os - implant « offrant jusqu'à $30 \%$ de plus » par rapport aux autres implants non plateau de même longueur. Cette réduction de $30 \%$ de la longueur de l'implant choisi sera fortement appréciée dans le cas de hauteur limitée de la crête, permettant ainsi d'éviter une fenestration d'une des tables osseuses ou d'endommager les racines des dents adjacentes. De plus l'utilisation d'un implant court permet, une fois placé, de modifier légèrement son axe si nécessaire, grâce à sa faible longueur et à la flexibilité de l'os.
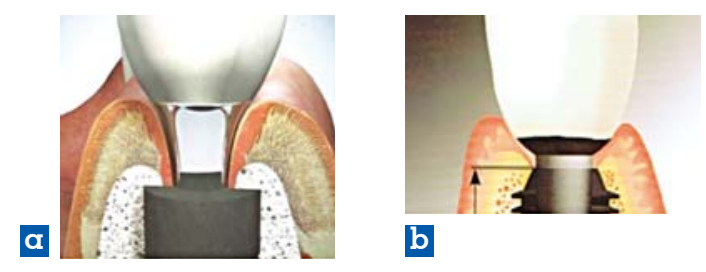

b

$\triangle$ Figure 10 :

(a) Plateforme sans épaulement oblique (Ankylos). (b) Plateforme avec épaulement oblique (Bicon).

$\triangle$ Figure 11 :

(a) Design des spires.

(b) Os cortical

(Bicon site).
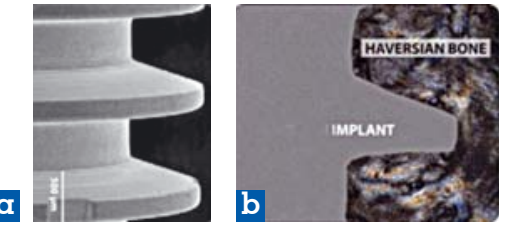
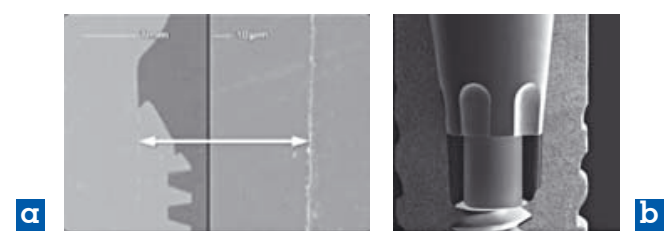

$\triangle$ Figure 12 :

(a) Taper Lock (Bicon). (b) Cone Morse (Ankylose).

Autre avantage, l'espace large entre les spires permet la régénération de l'os à partir du caillot sanguin avec un rythme plus rapide par rapport à l'os appositionnel qui se forme autour des implants ayant un design différent [32]. Cette caractéristique peut expliquer le succès de ces implants lorsqu'ils sont utilisés dans une situation difficile ou avec un os de qualité médiocre ou lors d'un contact intime avec les dents adjacentes.

\section{Interface pilier-implant (fig. 12)}

Avec le blocage conique, merveille de la technologie moderne, une soudure à froid se produit lorsque deux métaux similaires sont forcés ensemble de telle sorte qu'un échange d'atomes se produit entre eux. Cette liaison lorsqu'elle est faite avec suffisamment de précision (microgap de moins de 0,5 micron) assure un joint hermétique empêchant les infiltrations des germes bactériens au niveau de l'interface implant-pilier. Ces germes sont à l'origine de la résorption osseuse [35], ce qui explique son placement très profond jusqu'à $4 \mathrm{~mm}$ subcrestal sans conséquences inflammatoires (fig. 11). Les systèmes d'implants qui ne peuvent pas garantir une telle fermeture hermétique recommandent de placer leur implant au niveau de la crête afin de contrôler l'hygiène par un brossage efficace autour de l'implant. Les fuites microbiennes pourront provoquer des poches osseuses et même des péri-implantites avec pour conséquence la perte de l'implant.

\section{Taille de l'implant}

La dimension de l'implant est un paramètre important. L'utilisation des implants courts permet d'éviter une fenestration de la table osseuse, une lésion traumatique des racines des dents adjacentes ou une lésion des structures anatomiques importantes.

En cas d'implantation immédiate, le diamètre de l'implant doit être inférieur à celui de la dent. L'espace restant va être géré de la manière suivante :

I Si les parois osseuses sont intactes et si le défaut marginal péri-implantaire est de $3 \mathrm{~mm}$ ou moins, c'est seulement l'os autogène récolté et collecté qui sera utilisé pour combler ces lacunes afin de favoriser la régénération osseuse et de raccourcir la période de guérison. 
I Si l'espace péri-implantaire est de plus de $3 \mathrm{~mm}$, l'utilisation de l'os autogène récolté sera mis directement sur l'implant suivie par une matière de remplissage à base du B-Tricalcium phosphate. Ainsi le contour de la table externe sera maintenu et l'ampleur de la résorption osseuse réduite, tant horizontalement que verticalement $[42,43]$.

Habituellement, nous utilisons l'Easy-Graft Classique (B-Tricalcium phospate) pour sa manipulation facile car il peut être maintenu dans la cavité sans aucune protection type collagène ou fermeture de la plaie opératoire par première intention (cas 3). Les xénogreffes sont préconisées par opposition aux greffes osseuses autogènes, car elles se résorbent lentement et maintiennent le volume osseux stable dans le temps [43]. En cas d'implantation différée, si la dent a été extraite depuis 6 semaines ou plus, l'implant doit être placé de sorte que l'épaisseur osseuse soit de 1.5 à $2 \mathrm{~mm}$.

\section{Nombre d'implants}

Une sélection impropre du nombre d'implants peut provoquer un désastre esthétique (fig. 13), ou le contraire (cas 4 , fig. 7 ).
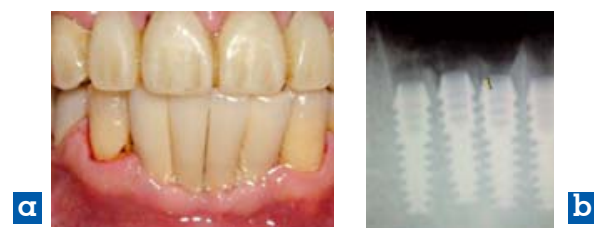

$\triangle$ Figure 13:

Désastre esthétique dû à un excès du nombre d'implants.

\section{APPROCHE ET T ECHNIQUE CHIRURGICALE (APPRÉCIATION MANUELLE DE L'OS)}

\section{Approche}

Les techniques implantation immédiate ou différée, en un ou deux temps, avec ou sans restauration immédiate, sont des approches chirurgicales différentes dont la sélection dépendra de plusieurs facteurs dont l'un d'entre eux est la compétence du chirurgien. Les autres facteurs peuvent être déterminés par un examen d'évaluation préopératoire approfondi des tissus durs et mous. L'image en trois dimensions, même pour les cas de dents unitaires, sera très appréciée afin d'éviter toute surprise peropératoire (fig. 2b).

Le biotype peux influencer le choix du protocole chirurgical, exemple un biotype gingival mince exige un protocole en deux temps avec une orientation particulière de l'implant (profond avec un axe d'insertion plus palatin) afin de favoriser la formation de muqueuse kératinisée [2] (Cas 1).

\section{Technique chirurgicale}

L'absence ou le déficit de l'os alvéolaire est un phénomène courant lié à plusieurs facteurs, anatomique, congénital, traumatique, pathologique, iatrogène, etc. La restauration esthétique optimale de l'implant dépendra de son bon positionnement tridimensionnel $[2,21,22,24]$.

Essayer de placer l'implant dans un site osseux défectueux sans reconstruction osseuse conduit souvent à la récession gingivale avec exposition de la zone cervicale de l'implant [2].

Autrement dit, en cas de déficit osseux, un résultat esthétique idéal est difficile à atteindre sans la préparation du site implantaire pré- ou peropératoire afin de pouvoir positionner l'implant dans les trois dimensions précises. Ce concept est connu sous le nom de positionnement guidé par la future émergence de la couronne.

Pour un résultat esthétique optimal, le point d'émergence de la future couronne guidera le chirurgien pour le positionnement exact de l'implant dans les 3 dimensions (fig. 1). En cas de carence des tissus durs et/ou mous, les procédures d'aménagement du site par greffe osseuse et/ou gingivale, ou par la régénération osseuse guidée ou autre doivent être effectuées en pré- ou peropératoire. Malheureusement, ces procédures sont parfois difficilement acceptées par les patients, elles sont plus traumatisantes, plus douloureuses et plus coûteuses.

Un autre concept opératoire qui est rarement pris en considération, qui semble être moins traumatisant et plus facilement accepté par les patients, est le placement guidé par l'os disponible. Dans ce concept, le chirurgien tente de placer son implant en fonction de la disponibilité osseuse, mais cette technique exige du praticien une bonne compétence chirurgicale. Le patient doit être informé de tous les avantages et inconvénients de chaque technique, avec leurs conséquences et leurs résultats finaux.

Pose d'implant immédiate (Cas 1, 3, 4). Le concept de la mise en place immédiate d'un implant est le protocole de choix, même lorsque la dent présente une pathologie péri-apicale chronique [20,37]. Il a été démontré que le placement immédiat de l'implant dans les sites d'extraction récente peut être une thérapie efficace, non seulement parce qu'elle réduit le nombre d'interférences chirurgicales « moins traumatique avec période de cicatrisation bien réduite » mais aussi parce 

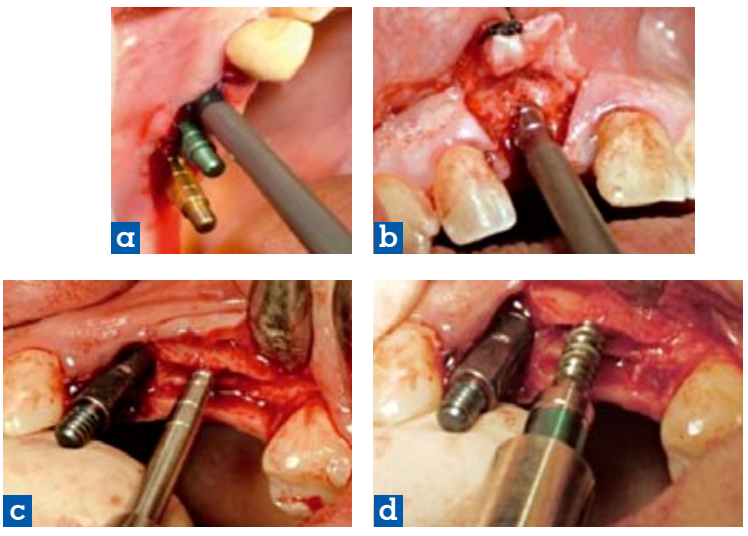

$\triangle$ Figure 14 :

(a) Ciseaux d'expansion. (c) Expansion osseuse. (d) Expansion à pas de vis.
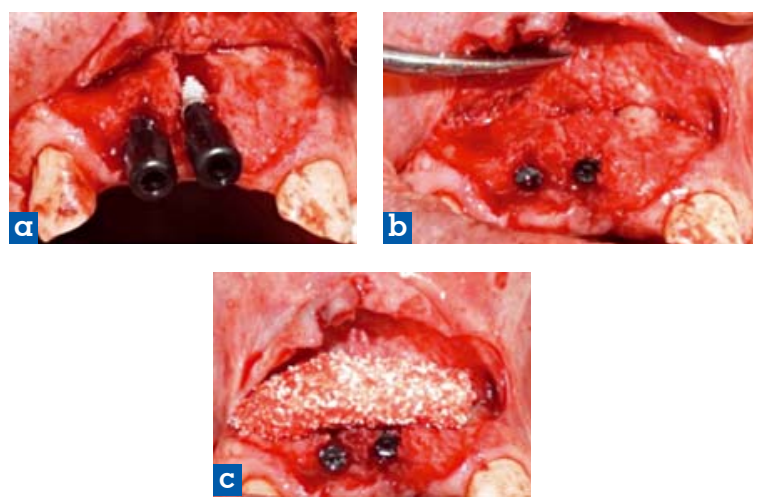

$\triangle$ Figure 15 :

MatriBone de Biomup. Mise en place de la greffe osseuse autogène directement sur l'implant. (b) Recouverte avec une xénogreffe. (c) Substitut osseux à base de céramique poreuse dispersée dans une matrice de collagène résorbable conférant des propriétés hémostatiques et ostéoconductrices.

qu'il favorise la préservation du contour de la table osseuse $[4,13,27,33]$.

Cependant, d'autres études vont plus loin et considèrent que l'implantation immédiate peut prévenir la résorption osseuse [38], tandis que d'autres auteurs ont suggéré que le placement immédiat de l'implant dans un site infecté est contre-indiqué [40, 41].

\section{Technique (Cas 1 et 4$)$}

Après une extraction dentaire la moins traumatisante possible et un curetage radical d'une lésion pathologique, l'inspection des parois osseuses doit être effectuée afin de déterminer les modalité de la technique. Un lambeau mucopériosté d'épaisseur totale n'est pas soulevé afin de préserver la vascularisation et d'éviter la perte osseuse ultérieure [16]. Cependant, ce lambeau est d'une grande valeur dans certaines situations car il assure une élimination complète de la lésion infectée, le contrôle en vision directe de notre ostéotomie, et enfin il facilite la mise en place d'un matériau de greffe (Cas 4).
Avec le foret pilote, on détermine la profondeur la plus adéquate de limplant, ainsi que son orientation en fonction de l'os disponible, puis des séquences manuelles de préparation de la loge en utilisant uniquement les alésoirs à main sans irrigation. Cette technique manuelle donne au chirurgien une excellente occasion d'apprécier la quantité, la qualité ou l'élasticité de l'os, ce qui pourrait être d'une grande utilité dans lecas d'un certain degré de déficience osseuse. Ici, la compétence du chirurgien sera l'acteur principal, qui peut exploiter cette fonctionnalité et modifier le volume osseux. De nombreuses techniques de manipulation de la crête (expansion et/ou de clivage en 1 ou 2 étapes) ont été appliquées pour résoudre ce déficit osseux (fig. 14) combinées ou non avec greffe autogène ou xénogreffe qui semblent être de grande valeur (fig. 15).

De plus, grâce à ces alésoirs manuels (de 1/4 côté coupant), nous pouvons non seulement récolter de l'os autogène, mais le récolter d'une manière sélective uniquement du côté où l'os est épais en évitant ainsi les parois minces (fig. 4h).

Nous appelons cette technique d'ostéotomie Ostéotomie d'appréciation manuelle (OAM) ou Esthétique Ostéotomie. Elle représente $90 \%$ de nos ostéotomies au niveau du maxillaire supérieur.

\section{POSITION ET ORIENTATION DE L'IMPLANT}

Après avoir sélectionné l'implant le plus adéquat (voir dimension de l'implant), il sera positionné en fonction de l'os disponible. Dans tous les types d'os, nous recommandons la mise en place de l'implant au moins à 1-2 mm en subcrestal et non pas au niveau de la crête. Nous considérons que la mise en place de l'implant au niveau de la crête est une situation à risque à moins que nous ayons un biotype gingival épais avec une ligne de sourire basse $[14,15]$.

\section{RESTAURATION PROTHÉTIQUE (PCI)}

La solution prothétique doit être adaptée à la position particulière de l'implant. Placer un implant dans une position si profonde pourrait résoudre la majorité des problèmes esthétiques, à condition de respecter les éléments suivants :

$\checkmark$ une parfaite étanchéité de l'interface implant-pilier prothétique avec absence totale de fuite microbienne; $\checkmark$ une gamme suffisante de piliers prothétiques avec hauteurs et angulations variables qui peut être adaptée aux positions de l'implant (voir fig. 4o, et p) ; 


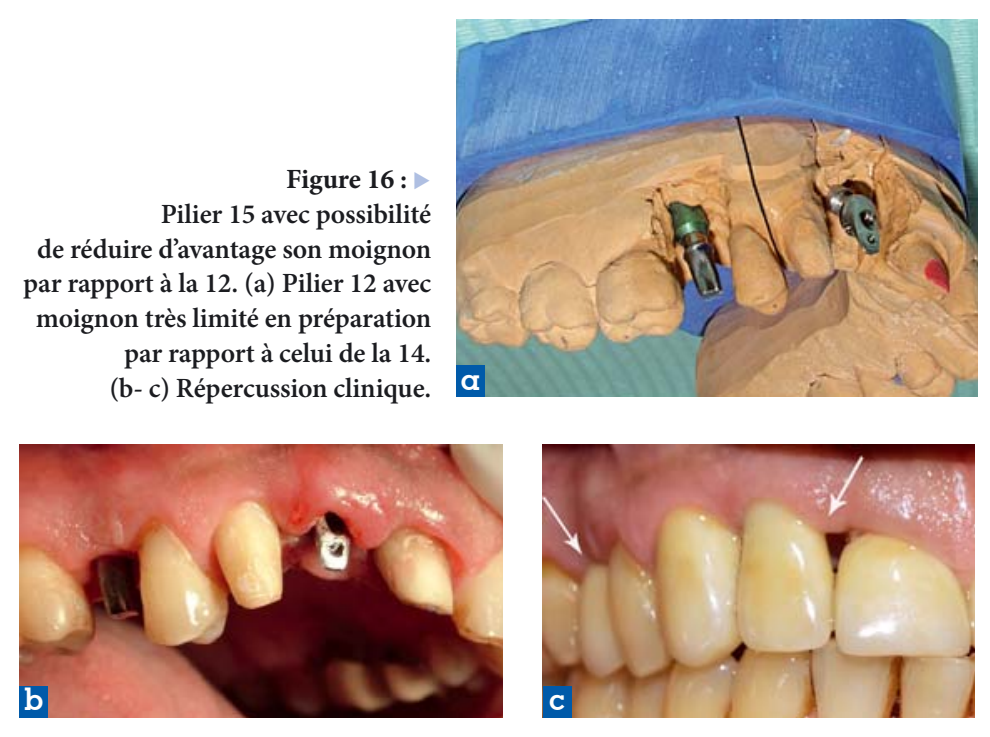

I flexibilité de modification du pilier prothétique ou sa réduction durant la réalisation de la future couronne (voir fig. 16a, b, c) ;

I fixation très rigide et hermétique de la couronne avec absence totale des débris de ciment qui agissent comme un corps étranger ;

$\checkmark$ l'utilisation de pilier à couronne intégrée (PCI) est une excellente solution, surtout dans des situations très critiques et difficiles à résoudre comme l'angulation forte jusqu'à 30-40\%. Elle est faite de composite à haute résistance fusionné directement au pilier durant sa réalisation au laboratoire (fig. 4o).

\section{CONCLUSION}

Les résultats que nous avons obtenus ces cinq dernières années auprès de 60 patients avec l'utilisation de 98 implants, donnant un taux de survie de 95,9\% reposent sur :

I la sélection de l'implant en termes de taille, macro-géométrie et nombre ;

$\checkmark$ une position sous-crestale avec une orientation de l'implant adaptée en fonction de l'os disponible ;

$\checkmark$ une approche chirurgicale adéquate avec une procédure manuelle (la moins traumatisante pour le tissu osseux) qui donne au chirurgien l'occasion d'évaluer, de manipuler et de modifier la structure osseuse, bien déterminée au cours de l'intervention ;

$\checkmark$ la restauration prothétique (en particulier la couronne à pilier intégré, CPI) avec une connexion (pilier-implant) parfaitement étanche et hermétique. Ce sont les éléments essentiels qui représentent les quatre piliers de l'implantologie et qui interagissent ensemble afin d'atteindre cet objectif. C'est notre Concept Esthétique et non Invasif de la zone Maxillaire antérieure.

Nous considérons que l'implant placé au niveau de la crête osseuse est une situation à risque, en particulier en cas de présence de micro gap (fuite microbienne) et de biotype gingival mince. Cette situation se traduira ultérieurement par une résorption de la crête osseuse avec toutes les conséquences inesthétiques et notamment une vision par transparence de la coloration métallique de l'implant.

\section{Bibliographie}

[1] Belser UC, Schmid B, Higginbottom F, et al. Outcome analysis of implant restorations located in the anterior maxilla: a review of the recent literature. Int J Oral Maxillofac Implants 2004;19(Suppl):30-42.

[2] Buser D, Martin W, Belser UC. Optimizing esthetics for implant restorations in the anterior maxilla: anatomic and surgical considerations. Int J Oral Maxillofac Implants 2004;19(Suppl):43-61.

[3] Buser, Chen, Weber, Belser. The concept of Early Implant Placement following Single Tooth Extraction in the Esthetic Zone. Biologic Rational and Surgical Procedures. Int J Periodont Rest Dent 2008;28:440-451.

[4] Belser U, Buser D, Higginbottom F. Consensus statements and recommended clinical procedures regarding esthetics in implant dentistry. Int J Oral Maxillofac Implants 2004;19(Suppl):73-4.
[5] Al-Sabbagh M. Implants in the esthetic zone. Dent Clin North Am 2006;50(3):391-407.

[6] Saadoun AP, Le Gall MG. Periodontal implications in implant treatment planning for aesthetic results. Pract Periodontics Aesthet Dent 1998;10(5):655-664.

[7] Carrion JB, Barbosa IR. Single implantsupported restorations in the anterior maxilla. Int J Periodontics Restorative Dent 2005;25(2):149-155.

[8] Jansen CE, Weisgold A. Presurgical treatment planning for the anterior single-tooth implant restoration. Compend Contin Educ Dent 1995;6(8):746, 748-752, 754.

[9] Kois JC. Predictable single tooth periimplant esthetics: five diagnostic keys. Compend Contin Educ Dent 2001;22(3):199-206.

[10] Kan JY, Rungcharassaeng K, Umezu K, Kois JC. Dimensions of peri-implant mucosa: an evaluation of maxillary anterior single implants in humans. J Periodontol 2003;74(4):557-62.
[11] Kan JY, Rungcharassaeng K, Umezu K, Kois JC. Dimensions of peri-implant mucosa: An evaluation of maxillary anterior single implants in humans. J Periodontol 2003;4:557-562.

[12] Kois JC. Predictable single tooth periimplant esthetics: Five diagnostic keys. Compend Contin Educ Dent 2001;22:199-206.

[13] Schwartz-Arad D, Chaushu G. Immediate implant placement: a procedure without incision. J Periodontol 1998;69:743-750.

[14] Ferrus J, Cecchinato D, Pjetrusson EB, Lang N, Sanz M, Lindhe J. Factors influencing immediate implant placement into extraction sockets. Clin Oral Impl Res 2010;21:22-29.

[15] Braut V, Bornstein MM, Belser U, Buser D. Thickness of the anterior maxillary facial bone wall-A retrospective study using cone beam computed tomography (CBCT). Int J Periodentics \& Restorative Dent 2011;31:125-131. 


\section{Bibliographie}

[16] Pennel BM, King KO, Wilderman MN, Barron JM. Repair of the alveolar process following osseous surgery. J Periodontol 1967;38:426-431.

[17] Berglundh T, Lindhe J. Dimension of the periimplant mucosa. Biological width revisited. J Clin Periodontol 1996;23:971-973.

[18] Cochran DL, Hermann JS, Schenk RK, Higginbottom FL, Buser D. Biologic width around titanium implants. A histo-metric analysis of the implanto-gingival junction around unloaded and loaded nonsubmerged implants in the canine mandible. J Periodontol 1997;68:186-198.

[19] Todescan FF, Pustiglioni FE, Imbronito $\mathrm{AV}$, Albrektsson T, Gioso M. Influence of the microgap in the peri-implant hard and soft tissues: A histomorphometric study in dogs. Int J Oral Maxillofac Implants 2002;17:467-472.

[20] Siegenthaler DW, Jung RE, Hoderegger C, Roos M, Hammerle CHF. Replacement of teeth exhibiting periapical pathology by immediate implants. A prospective controlled clinical trial. Clin Oral Impl Res 2007;18:727-737.

[21] Garber DA, Belser UC. Restoration-driven implant placement with restoration-generated site development. Compend Contin Educ Dent 1995;16(8):796, 798-802, 804.

[22] Garber DA. The esthetic dental implant: letting restoration be the guide. J Am Dent Assoc 1995;126(3):319-325.

[23] Byrne G. Platform Switching reduces peri-implant bone loss. J Am Dent Assoc 2012;143:787-788.

[24] Higginbottom FL, Wilson TG Jr. Threedimensional templates for placement of root-form dental implants: a technical note. Int $J$ Oral Maxillofac Implants 1996;11(6):787-793.
[25] Lazzara RJ, Porter SS. Platform switching: A new concept in implant dentistry for controlling postrestorative crestal bone levels. Int J Periodont Restorat Dent 2006;26:9-17.

[26] Baumgarten H, Cocchetto R, Testori T, Meltzer A, Porter S. A new implant design for crestal bone preservation: Initial observations and case report. Pract Proced Aesthet Dent 2005;17:735-740.

[27] Gardner DM. Platform switching as a means to achieving implant esthetics. N Y State Dent J 2005;71:34-37.

[28] Prasad KD, Shetty M, Bansal N, Hegde C. Platform switching: An answer to crestal bone loss. J Dental Implants 2001;1(1):13-17.

[29] Prosper L, Redaelli S, Pasi M, Zarone F, Radaelli G, Gherlone EF. A randomized prospective multicenter trial evaluating the platform-switching technique for the prevention of postrestorative crestal bone loss. Int J Oral Maxillofac Implants 2009;24(2):299-308.

[30] Hirzeler M, Fickl S, Zuhr O, Wachtel HC. Peri-implant bone level around implants with platform-switched abutments: Preliminary data from a prospective study. J Oral Maxillofac Surg 2007;65:33-39.

[31] Degidi M, Iezzi G, Scarano A, Piattelli A. Immediately loaded titanium implant with a tissue-stabilizing/maintaining design ('beyond platform switch') retrieved from man after 4 weeks: A histological and histomorphometrical evaluation: A case report. Clin Oral Implants Res 2008;19:276-282.

[32] Lemons JE. Biomaterials, Biomechanics, Tissue Healing, and Immediate-Function Dental Implants. J Oral Implantology 2004;30(5):318-324.

[33] Lazzara RJ. Immediate implant placement into extraction sites: surgical and restorative advantages. Int J Periodontics Restorative Dent 1989;9:333-343

[34] Grunder U. Stability of the mucosa topography around single-tooth implants and adjacent teeth: 1-year results. Int $J$ Periodontics Restorative Dent 2000;20:11-17.
[35] Broggini N, et al. Peri-implant Inflammation defined by the implant-abutment interface. $J$ Dent Res 2006;85(5):473-478.

[36] Polizzi G, et al. Immediate and delayed implant placement into extraction sockets: a 5-year report. Clin Implant Dent Relat Res 2000;2:93-99.

[37] Del Fabbro M, Boggian C, Taschieri S. Immediate implant placement into fresh extraction sites with chronic periapical pathologic features combined with plasma rich in growth factors: Preliminary results of single-cohort study. J Oral Maxillofac Surg 2009;67:2476-484.

[38] Botticelli D, BerglundhT , Lindhe J. Hardtissue alterations following immediate implant placement in extraction sites. J Clin Periodontol 2004;31:820-828.

[39] Araujo M, Wennstrom JL, Lindhe J. Modeling of the buccal and lingual bone walls of fresh extraction sites following implant installation. Clin Oral Impl Res 2006;17:606-614.

[40] Novaes AB Jr, Marcaccini AM, Souza SL, Taba M Jr, Grisi MF. Immediate placement of implants into periodontally infected sites in dogs: a histomorphometric study of bone-implant contact. Int J Oral Maxillofac Implants 2003;18(3):391-398.

[41] Waasdorp JA, Evian CI, Mandracchia M. Immediate placement of implants into infected sites: a systematic review of the literature. J Periodontol 2010;81(6):801-808.

[42] Capellia M, Testorib T, Gallic F, Zuffettid F, Del Fabbroe M. Esthetic risk assessment of immediate implant: position paper and proposal of a novel diagnostic parameter. Italian Oral Surgery 2012;11(5):158-174.

[43] Baglivo M, Campos H, Carrasco MA, Pascual A, Levi P, Nart J. Ridge preservation and GTR with a xenograft and resorbable collagen membrane. Spain Implants 2011;3:14-18.

[44] Langer B, Calagna L. The subepithelial connective tissue graft. J Prosthet Dent 1980; 44(4):363-7.

\section{edpdentaire \\ La référence du monde dentaire}

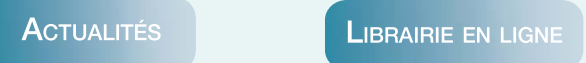

Retrouvez les petites annonces sur le portail www.edp-dentaire.fr

ARTICLES

CLINIQU
Produits

Petites

ANNONCES 